\title{
Equine Fetal, Adult, and Embryonic Stem Cell-Derived Tenocytes Are All Immune Privileged but Exhibit Different Immune Suppressive Properties In Vitro
}

\author{
Alyce McClellan, ${ }^{1}$ Yasmin Z. Paterson,, ${ }^{1,2}$ Romain Paillot, ${ }^{3,4}$ and Deborah Jane Guest ${ }^{1}$
}

In horses and humans, tendon injuries are a significant problem. Not only can they occur in both athletes and nonathletes, they require lengthy periods of recuperation and undergo poor natural regeneration, which leads to high reinjury rates. Embryonic stem cells (ESCs) may provide a renewable source of allogeneic cells to use in clinical applications to aid tissue regeneration. Equine ESCs can undergo tenocyte differentiation in vivo and in vitro, but the immune properties of tenocytes isolated from either ESCs or tissues have not previously been characterized. Here, we demonstrate that equine tenocytes derived from fetal and adult tendon tissue and ESCs express robust levels of major histocompatibility complex (MHC) I but no MHC II in response to inflammatory cytokine interferon gamma (IFN $\gamma$ ). However, MHC expression does not affect their allorecognition by peripheral blood mononuclear cells in vitro. Adult and fetal tenocytes remain immune privileged and strongly immune suppressive in both the presence and absence of exogenously applied IFN $\gamma$. In contrast, ESC-derived tenocytes are immune privileged even in the presence of IFN $\gamma$, but they are only weakly immune suppressive in the presence but not in the absence of exogenously applied IFN $\gamma$. This is despite ESC-tenocytes expressing a number of genes involved in immune modulation at significantly higher levels than those expressed by adult and fetal tenocytes when in standard, nonstimulated monolayer culture. Together, this work suggests that, similar to other fibroblasts, tenocytes have immune modulatory properties, and that culture-expanded tenocytes derived from primary tissues or ESCs may be safe to use in clinical transplantations to injured tendons of unrelated animals.

Keywords: tenocytes, embryonic stem cells, immunogenicity, fetal, immune suppression

\section{Introduction}

$\mathrm{T}$ ENDON INJURIES OCCUR commonly in human and equine athletes, accounting for $46 \%$ of all limb injuries in racehorses [1]. They also occur in nonathletes and are estimated to comprise $30 \%-50 \%$ of all musculoskeletal injuries in humans [2]. The structure and function of tendons are very similar in horses and humans, and they share many of the same risk factors for tendon injuries such as age and training. Horses may therefore provide a relevant large animal model for studying both the human disease process and evaluating novel therapies $[3,4]$.

Tendon injuries in both humans and horses heal through the formation of scar tissue, which is biomechanically inferior to healthy tendon tissue, and this predisposes them to a high risk of reinjury (up to $67 \%$ in racehorses [5]). Tendon injuries are therefore the number one reason for retirement from racing [6] and have a significant welfare impact on the equine industry. Tendon injuries are also a leading cause of morbidity in humans.
In contrast to adult tendon injuries, fetal tendon injuries regenerate without scar tissue formation [7] in a process that is intrinsic to the tendon cells themselves [8,9]. Reducing scar tissue formation after an adult tendon injury and increasing the regeneration of healthy tendon tissue would reduce the frequency of reinjury, and many regenerative medicine strategies for tendon injuries are being investigated. Adult tendon autografts can be performed but are associated with donor site morbidity [10]. Allogeneic adult tendon tissue transplantations can also be performed [11], but the tissues require decellularization to remove the residing immune cells [12] and are therefore only providing a new scaffold to aid endogenous cell regeneration. Adult and fetal tendon cells (tenocytes) themselves have been used in autologous transplantations, with fetal tenocytes giving improved tissue regeneration compared with adult cells in a mouse model [9]. However, as it is difficult to get sufficient numbers of allogeneic cells for human or equine clinical application, it remains unknown if adult and/or fetal

\footnotetext{
${ }^{1}$ Centre for Preventive Medicine, Animal Health Trust, Newmarket, United Kingdom.

${ }^{2}$ Department of Veterinary Medicine, University of Cambridge, Cambridge, United Kingdom.

${ }^{3}$ LABÉO Frank Duncombe, Caen, France.

${ }^{4}$ Normandie University, UniCaen, Biotargen, Saint-Contest, France.
} 
tenocytes could be used safely in allogeneic transplantations or if they would generate an immune response. To overcome the limitation with the supply of allogeneic tenocytes, embryonic stem cells (ESCs) may provide a source of cells for clinical use.

We have previously derived and characterized equine ESCs [13,14]. These cells can be grown indefinitely in culture while retaining the ability to differentiate into derivatives of all three germ layers (endoderm, ectoderm, and mesoderm), which opens up the potential of using them in regenerative medicine strategies. We have previously demonstrated that in vivo equine ESCs exhibit a high survival in the injured tendon and express the tendon-associated transcription factor scleraxis $[15,16]$. This is in stark contrast to mesenchymal stromal cells (MSCs), which have a very poor survival rate in the injured tendon $[15,17]$. In vitro ESCs can differentiate into tenocytes in response to $3 \mathrm{D}$ culture and/or transforming growth factor beta 3 (TGF- $\beta 3$ ) $[16,18]$ in a process that is dependent on scleraxis [19].

As ESCs, by definition, can only be used in allogeneic transplantation, the immunogenicity of the cells and their differentiated derivatives must be defined. In the injured tendon in vivo, equine ESCs survived in high numbers without any signs of a cell-mediated immune response [15]. However, the maximum duration of this study was only 3 months, and the longer term survival/rejection of the cells is unknown. In vitro studies further demonstrated that equine ESCs failed to induce the proliferation of allogeneic peripheral blood mononuclear cells (PBMCs), either in their undifferentiated state or after spontaneous, nondirected differentiation into a mixed variety of cell types [20].

However, the immunogenicity of ESCs after tenocyte differentiation has not yet been reported. In fact, although immunogenicity of equine and human MSCs is well studied [20-25], to our knowledge there are no studies on the immunogenicity of normal tenocytes isolated from healthy tendon tissue from either species. Investigations into cellular properties and behavior must take into account any impact the inflamed tissue environment might have on the transplanted cells. There are high levels of inflammation in the acutely injured tendon [26-30] with inflammatory cytokines interferon gamma (IFN $\gamma)$, tumor necrosis factor alpha (TNF $\alpha)$, and interleukin 1 beta (IL-1 $\beta$ ) being present $[31,32]$. It is therefore important to understand how any cells that are applied clinically in therapeutic strategies respond to the inflammation that is present.

Recently, we have demonstrated that while inflammatory cytokine IL-1 $\beta$ has negative effects on adult and fetal equine tenocyte gene expression and collagen contraction, ESCderived tenocytes are protected from these effects due to their differential expression of IL- $1 \beta$ receptors and antagonists [33]. We have previously shown that IFN $\gamma$ increased major histocompatibility complex (MHC) I (but not MHC II) expression in undifferentiated and spontaneously differentiated ESCs, but the cells still failed to induce the proliferation of allogeneic PBMCs [20].

In addition to a cell's ability to induce an immune response, in vitro cocultures are often used to determine the ability of cells to suppress the proliferation of activated PBMCs. It has been widely shown that human and equine MSCs are able to suppress PBMC proliferation and modulate the expression of inflammatory cytokines by PBMCs through their expression of a variety of secreted factors $[20,22,25]$. Immune modulation is a common feature of MSCs isolated from multiple species [34] and is believed to underpin the mechanisms by which they can enhance tissue regeneration $[35,36]$. However, there are reports in the literature that rather than this being an MSC-specific property, it is actually a property of many types of fibroblasts [37-43].

In this study, we determined the immunogenicity and immune suppressive properties of equine tenocytes isolated from ESCs, adult and fetal tendon tissue in the presence and absence of inflammatory cytokines to determine whether they are likely to be safe in future in vivo allogeneic transplantations.

\section{Materials and Methods}

\section{Tenocyte isolation and culture}

All tendon tissue samples were taken with the approval of the Animal Health Trust Ethical Review Committee (AHT02_ 2012). Tenocytes were isolated postmortem from healthy tendons of three adult Thoroughbred horses that had been euthanized for reasons unrelated to this project, and from fetal tendons of three fetuses at 319, 320, and 321 days of gestation, which had undergone spontaneous abortion.

Tenocytes were isolated and cultured as described previously [16]. In brief, the tendon tissue was dissected into small pieces and incubated in $1 \mathrm{mg} / \mathrm{mL}$ type I collagenase (SigmaAldrich, Dorset, United Kingdom) overnight at $37^{\circ} \mathrm{C}$. Dissociated cells were cultured in Dulbecco's modified Eagle's medium (DMEM) high glucose, $10 \%$ fetal bovine serum, $2 \mathrm{mM}$ L-glutamine, $100 \mathrm{U} / \mathrm{mL}$ penicillin, $100 \mu \mathrm{g} / \mathrm{mL}$ streptomycin (all from Invitrogen, Renfrewshire, United Kingdom) and passaged using $0.25 \%$ trypsin-EDTA (Sigma-Aldrich) every 3-4 days. All tendon cells had been previously characterized for their tendon gene expression and ability to contract a collagen gel [18,19,33].

\section{ESC culture and differentiation}

ESCs from three different embryos were previously derived and characterized [14-16,18,19]. ESCs were cultured and differentiated in 2D as described previously [16]. Undifferentiated ESCs were cultured on mitotically inactivated mouse embryonic fibroblasts at $37^{\circ} \mathrm{C}, 5 \% \mathrm{CO}_{2}$ in $\mathrm{ESC}$ medium (DMEM/F12 containing $15 \%$ fetal bovine serum, $2 \mathrm{mM}$ L-glutamine, $1 \%$ nonessential amino acids, $1 \mathrm{mM}$ sodium pyruvate, $0.1 \mathrm{mM} 2$-mercaptoethanol) (all from Invitrogen), and 1,000 U/mL leukemia inhibitory factor (LIF) (Sigma-Aldrich). ESCs were passaged mechanically every 5-7 days in the presence of $2 \mu \mathrm{M}$ thiazovivin (StemGent, Cambridge, MA). To differentiate the ESCs to tenocytes, they were cultured without feeders in ESC media lacking LIF and in the presence of $20 \mathrm{ng} / \mathrm{mL}$ TGF- $\beta 3$ (Peprotech, London, United Kingdom) for 14 days.

\section{Immunocytochemistry}

Three lines of adult and fetal tenocytes were plated onto gelatin-coated coverslips and treated with no cytokines (control), or $17 \mathrm{ng} / \mathrm{mL}$ IL-1 $\beta, 10 \mathrm{ng} / \mathrm{mL} \mathrm{TNF} \alpha$, and/or $100 \mathrm{ng} / \mathrm{mL}$ IFN $\gamma$ (all from Peprotech) either individually, or in combination for $72 \mathrm{~h}$ before the cells being used in immunocytochemistry. Adult and fetal tenocytes were used between 
passages 4 and 10. Three lines of ESC-tenocytes were differentiated from ESCs between passages 17 and 20 on gelatin-coated coverslips and treated either with no cytokines (control) or $100 \mathrm{ng} / \mathrm{mL}$ IFN $\gamma$. Cells were fixed in $3 \%$ paraformaldehyde for $20 \mathrm{~min}$, permeabilized in $0.1 \%$ Triton $\mathrm{X}-100$ for $1 \mathrm{~h}$, blocked in $2.5 \%$ normal horse serum for $20 \mathrm{~min}$, and incubated with primary antibodies overnight at $4^{\circ} \mathrm{C}$ before washing and detection with secondary antibodies. Primary antibodies were mouse anti-MHC I 1:200 dilution (H58A; VMRD, Oxfordshire, United Kingdom) and mouse anti-MHC II 1:100 (MCA1085GA; BioRad, Hertfordshire, United Kingdom). Secondary antibody was goat antimouse Alexa Fluor 594 (A11005; Invitrogen). As a positive control for MHC II staining fixed and permeabilized equine PBMCs were used.

\section{Isolation of PBMCs}

Blood was collected from Welsh Mountain ponies under a Home Office Licence (PPL 7007953) and with the approval of the Animal Health Trust Animal Welfare Ethical Review Board (RPP 02-14). PBMCs were purified by centrifugation on Ficoll-Hypaque (Amersham Biosciences, Uppsala, Sweden) as previously described [44]. Isolated PBMCs were suspended in $1 \mathrm{~mL}$ of PBMC media [RPMI-1630 (Sigma) with $10 \%$ heat-inactivated fetal calf serum, $100 \mathrm{U} / \mathrm{mL}$ penicillin and $100 \mu \mathrm{g} / \mathrm{mL}$ streptomycin, $2 \mathrm{mM}$ L-glutamine and $55 \mu \mathrm{M} 2$ mercaptoethanol] (all from Invitrogen) and counted.

\section{PBMC and tenocyte coculture}

These experiments were performed using three lines of adult, fetal, and ESC-derived tenocytes, each with one to three lines of PBMCs (derived from different animals), and each experiment was performed in triplicate. Fetal and adult tenocytes were used between passages 7 and 10 in these experiments. ESC-tenocytes were differentiated from ESCs between passages 15 and 29 for use in these experiments. Tenocytes were treated with $10 \mu \mathrm{g} / \mathrm{mL}$ mitomycin C (Sigma) for $2 \mathrm{~h}$ at $37^{\circ} \mathrm{C}$ before washing, trypsinization, and numerating. Tenocytes $\left(5 \times 10^{4}\right)$ were plated per well of a flat-bottomed 96-well plate and cultured with $2.5 \times 10^{5}$ allogenic effector PBMCs in PBMC media (ie, 1:5 ratio stimulator:effector cells). The cells were then cultured at $37^{\circ} \mathrm{C}, 5 \% \mathrm{CO}_{2}$ in either the absence (control) or the presence of $100 \mathrm{ng} / \mathrm{mL} \mathrm{IFN} \gamma$ for 4 days, before the addition of radioactive thymidine $\left({ }^{3} \mathrm{H}\right.$ thymidine) (GE Healthcare Biosciences) at a final concentration of $0.5 \mu \mathrm{Ci}$ per well and incubated at $37^{\circ} \mathrm{C}, 5 \% \mathrm{CO}_{2}$ for $16-18 \mathrm{~h}$ before freezing at $-20^{\circ} \mathrm{C}$. After thawing, the cells were harvested using a FilterMate (Perkin Elmer, Beaconsfield, United Kingdom) and counted using a TopCount NXT (Perkin Elmer). To provide positive and negative controls, PBMCs were cocultured with allogeneic and autologous PBMCs, respectively. In these experiments, the stimulator PBMCs were treated with $50 \mu \mathrm{g} / \mathrm{mL}$ mitomycin $\mathrm{C}$ for $30 \mathrm{~min}$ at $37^{\circ} \mathrm{C}$, before washing and numerating. Stimulatory PBMCs $\left(5 \times 10^{4}\right)$ were then cultured with $1 \times 10^{5}$ effector PBMCs in PBMC media in round-bottomed 96-well plates (ie, 1:2 ratio stimulator:effector cells) for 4 days before ${ }^{3} \mathrm{H}$ addition and harvesting as above. Student's $t$-test was used to determine a significantly different level of PBMC proliferation relative to nonactivated PBMCs.

\section{PBMC suppression experiments}

For culture stimulation and suppression experiments, fetal, adult, and ESC-derived tenocytes were mitotically inactivated with mitomycin $\mathrm{C}$ as described above, and $5 \times 10^{4}$ cells plated per well of a 96-well flat-bottomed plate. PBMCs $\left(2.5 \times 10^{5}\right.$ or $\left.1.25 \times 10^{5}\right)$ were added and stimulated to proliferate by treatment with $5 \mu \mathrm{g} / \mathrm{mL}$ phytohemagglutinin (PHA) (Sigma). This was performed in the presence or absence of $100 \mathrm{ng} / \mathrm{mL}$ IFN $\gamma$. These cultures were carried out for 4 days before ${ }^{3} \mathrm{H}$ addition and harvesting as above. To produce a baseline level of proliferation, PBMCs were treated with $5 \mu \mathrm{g} / \mathrm{mL}$ PHA in the absence of coculture with tenocytes. Student's $t$-test was used to determine a statistically significant level of inhibition.

\section{RNA sequencing}

Three lines (derived from three individual horses) of adult, fetal, and ESC-derived tenocytes cultured in 2D were used in these experiments. Fetal and adult tenocytes were used between passages 3 and 7. ESC-tenocytes were differentiated from ESCs between passages 18 and 22 for use in these experiments. RNA was extracted using Tri-reagent (Sigma), purified using an RNeasy mini Kit (Qiagen, Manchester, United Kingdom), and contaminating genomic DNA removed using Ambion DNA-free (Life Technologies, Paisley, United Kingdom). RNA concentration was measured using a Nanodrop (ThermoFisher, Loughborough, United Kingdom) and a Qubit (ThermoFisher). RNA integrity was confirmed on a Tapestation (Agilent, Milton Keynes, United Kingdom) by an external provider (Cambridge Genomics, Cambridge, United Kingdom). mRNA libraries were prepared using a TruSeq stranded mRNA kit (Illumina, Cambridge, United Kingdom), and RNA sequencing was performed on a NovaSeq6000 (Illumina) both by an external provider (Edinburgh Genomics, Edinburgh, United Kingdom). This generated 20.2-23.8 million reads of 100 base pair paired end data per sample. Resulting FASTQ files were quality control (QC) checked using FASTQC and FASTQ Screen (Babraham Bioinformatics, Cambridge, United Kingdom). QC-checked reads were aligned to the Ensemble version v95 EquCab 3.0 transcriptome using the pseudoaligner Salmon [45] in Quasimapping-based mode with GC-bias correction (-gcBias). The tximport package [46] was used to import the quantified genelevel abundance data into R (v.3.5.2), and differential expression analysis conducted using R/Bioconductor DSeq2 (v.1.22.2) as described in Love et al. [47]. Genes with adjusted $P$ value of $<0.01$ and $\log 2$-fold change of \pm 2 were considered as differentially expressed. The biological processes that differentially expressed genes were predicted to affect were identified using gene ontology software Panther GO-Slim (www.pantherdb.org).

\section{Results}

We have previously demonstrated that in 2D culture, equine ESCs can be induced to differentiate into tenocytes that exhibit a change in morphology and express tenocyte marker genes and proteins [16]. After 14 days of differentiation, $41 \%$ of cells express the mature tenocyte marker tenomodulin in comparison with $77 \%$ of adult tenocytes and $70 \%$ of fetal tenocytes [33]. 
IFN $\gamma$ increases MHC I but not MHC I/ expression by equine adult, fetal, and ESC-derived tenocytes

Adult and fetal tenocytes express no detectable MHC I protein in control 2D cultures, whereas ESC-tenocytes exhibit low, heterogeneous expression of MHC I. No MHC II protein is detected under normal conditions in adult, fetal, or ESC- tenocytes (Fig. 1A). Exposure of adult, fetal, and ESCtenocytes to $100 \mathrm{ng} / \mathrm{mL}$ IFN $\gamma$ for $72 \mathrm{~h}$ leads to the robust expression of MHC I protein but fails to induce the detectable expression of MHC II protein. In contrast, TNF $\alpha$ and IL-1 $\beta$, other cytokines commonly present in the injured tendon, did not induce the expression of MHC I or MHC II in fetal or adult tenocytes (and hence were not tested on ESC-tenocytes).
A
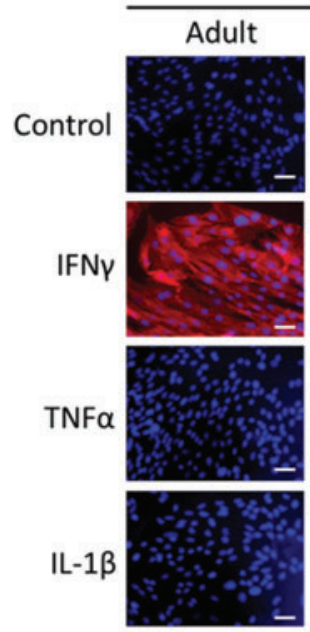

IFNY +

TNF $\alpha+$ IL-1 $\beta$
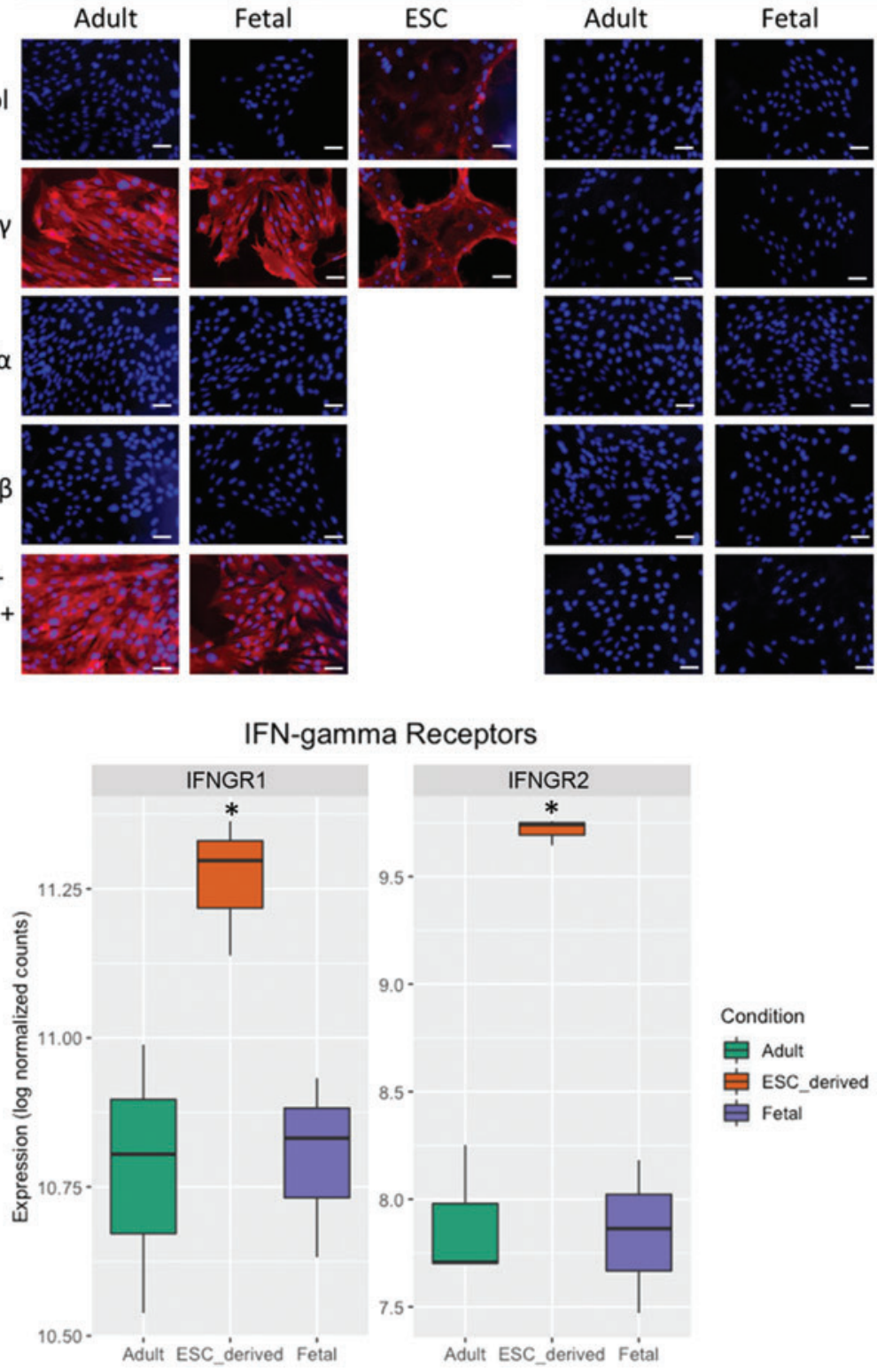

\section{B}

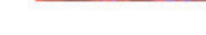

IFN-gamma Receptors
MHC II

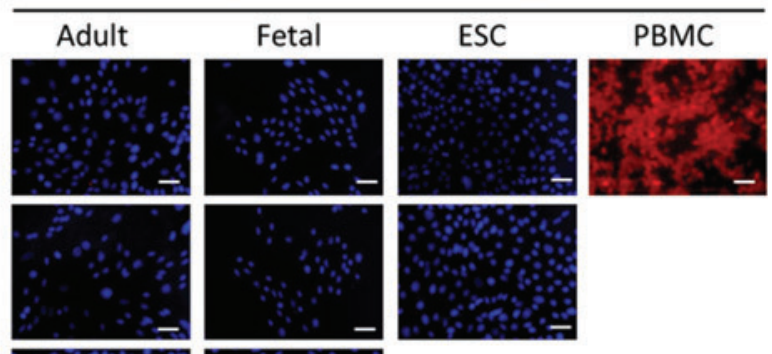


A combination of all three cytokines did not have any additive effect on MHC I expression compared with IFN $\gamma$ alone in adult and fetal tenocytes, and did not induce MHC II expression (Fig. 1A). RNA sequencing data demonstrated that adult, fetal, and ESC-tenocytes all express IFN $\gamma$ receptors, with ESC-tenocytes expressing significantly more IFNGRI and IFNGR2 than adult and fetal tenocytes (Fig. 1B).

\section{Equine tenocytes fail to induce the proliferation of allogeneic PBMCs in vitro even in the presence of IFNy}

Adult tenocytes (Fig. 2A), fetal tenocytes (Fig. 2B), and ESC-tenocytes (Fig. 2C) were mitotically inactivated and cocultured with allogeneic PBMCs in 2D in direct contact. No proliferation of the allogeneic PBMCs was observed with either adult, fetal, or ESC-tenocytes when cultured in either the absence (control) or the presence of $100 \mathrm{ng} / \mathrm{mL}$ IFN $\gamma$.

\section{Fetal and adult equine tenocytes suppress the proliferation of PHA-activated PBMCs to a high degree, but ESC-derived tenocytes only produce low levels of suppression}

We next determined if tenocytes from the three different sources were capable of suppressing the proliferation of ac- tivated PBMCs in direct coculture (Fig. 3). Adult and fetal tenocytes were found to significantly inhibit PHA-induced PBMC proliferation by $70 \%-80 \%$ when used at a ratio of $1: 5$, tenocytes:PBMCs, and this was not affected by the presence of IFN $\gamma$. In contrast, ESC-tenocytes failed to produce any suppression of PHA-activated PBMC proliferation, in the presence or absence, of IFN $\gamma$ when used at a 1:5 ratio. To account for the heterogeneity of ESC-tenocyte differentiation in 2D (where only $41 \%$ of cells express the mature tenocyte marker tenomodulin [33]), we increased the ratio of ESCtenocytes:PBMCs to $1: 2.5$, and found that this did produce a low level of suppression $(20 \%-30 \%)$, but this was only significant when the cells were in the presence of IFN $\gamma$.

\section{Genes associated with immune suppression vary in expression in adult and fetal tenocytes compared with ESC-tenocytes}

RNA sequencing was performed on adult tenocytes, fetal tenocytes, and ESC-tenocytes cultured in 2D. There were 1,691 genes that were upregulated in both fetal and adult tenocytes compared with ESC-tenocytes that had a $\log 2$-fold change of $\geq 2$ and an adjusted $P$ value of 0.01 (the full data set is available in Supplementary Table S1). Gene ontology analysis was performed to determine the biological pathways that were significantly over-represented by these
A

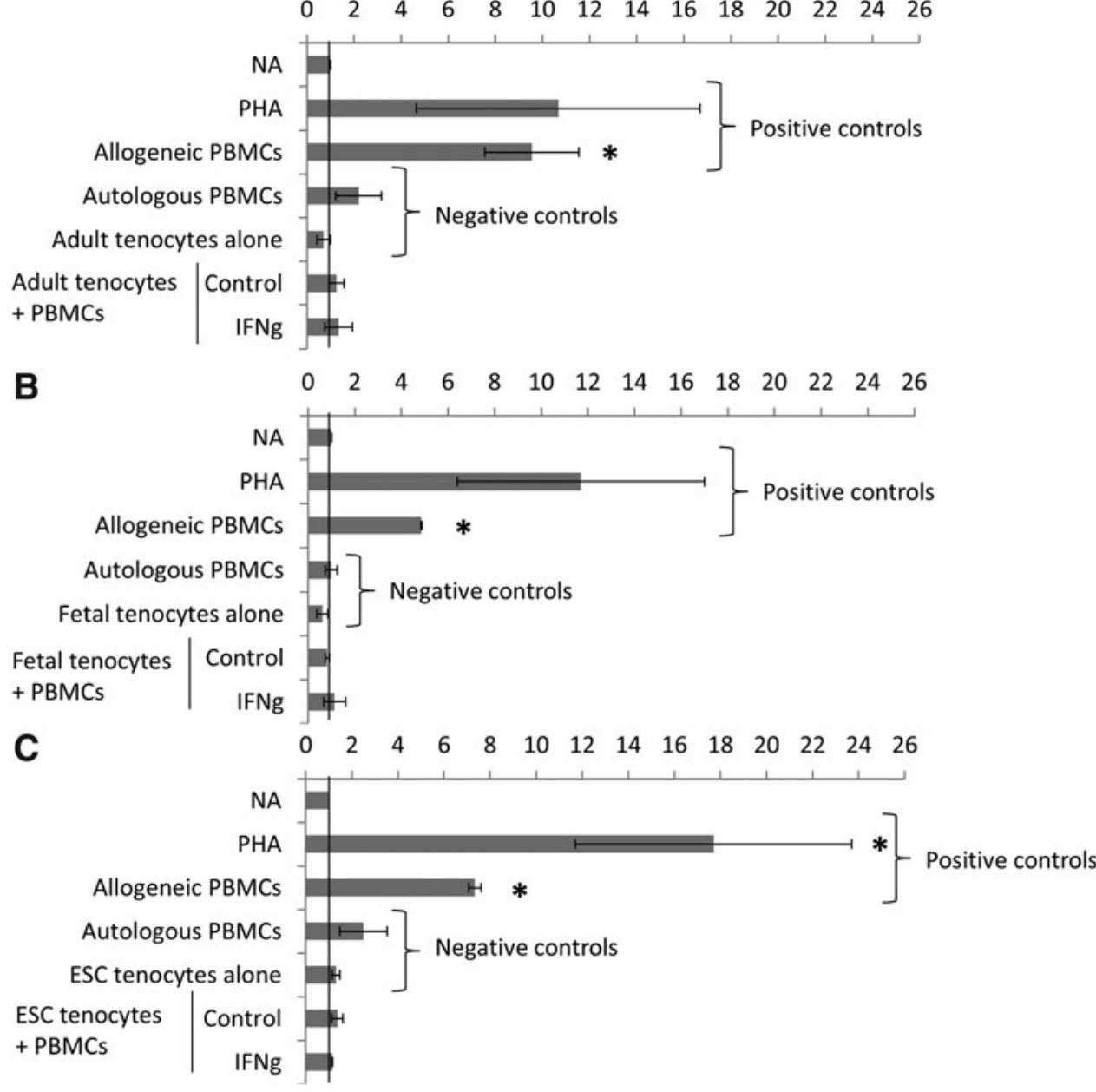

FIG. 2. Adult, fetal, and ESC-tenocytes do not induce the proliferation of allogeneic PBMCs in direct cocultures even in the presence of IFN $\gamma$. NA PMBCs were used as a background control. PMBCs were stimulated to proliferate with $5 \mu \mathrm{g} / \mathrm{mL}$ PHA, or were cultured with mitotically inactivated allogeneic PBMCs to induce proliferation and provide positive controls. Mitotically inactivated tenocytes (adult, fetal, or ESC derived) do not proliferate and were used in coculture with allogeneic PBMCs. PBMCs do not proliferate in response to coculture with allogeneic adult (A), fetal (B), or ESCtenocytes $(\mathbf{C})$ whether in the absence (control) or presence of IFN $\gamma$. Error bars represent the SEM of three biological replicates of tenocytes each cultured with PBMCs isolated from two different animals. Student's $t$-test was used to determine statistically significant differences in PBMC proliferation relative to NA $* P<0.05$. NA, nonactivated; PHA, phytohemagglutinin. 


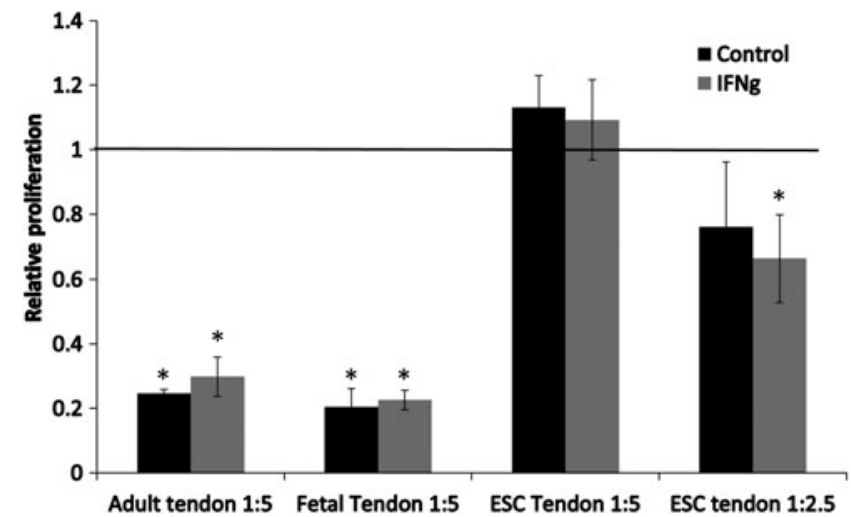

FIG. 3. Adult and fetal tenocytes, but not ESC-tenocytes, can significantly suppress the proliferation of PHA-activated PBMCs. Adult, fetal, and ESC-tenocytes were cultured with PHA-activated PBMCs at a ratio of 1:5 tenocytes to PBMCs. At this ratio, both adult and fetal tenocytes significantly suppress PBMC proliferation, but ESC-tenocytes do not produce any suppression, and this is not affected by IFN $\gamma$ being present (IFN $\gamma$ ) or absent (control). A low level of suppression is produced when ESC-tenocytes are used at a ratio of 1:2.5 with PBMCs, but this is only significant when IFN $\gamma$ is present. The relative proliferation compared with PHA stimulated PBMCs cultured alone is shown, which has been set a baseline value of 1.0 as indicated by the solid horizontal line. Error bars represent the SEM of three biological replicates of tenocytes each cultured with PBMCs isolated from two different animals. Student's $t$-test was used to determine statistically significant levels of PBMC suppression compared with the baseline $* P<0.05$.

genes. There were 81 biological pathways over-represented in the data set (Supplementary Table S2), and the top 15 most significant are shown in Fig. 4. Extracellular matrix organization (GO:0030198) and structure organization (GO:0043062) were the most significantly over-represented biological pathways, but pathways such as signal transduction (GO:0007165), cell surface receptor signaling pathway (GO:0007166), and response to stimulus (GO:0051716) were also over-represented.
Table 1. Genes with a Published Role in Mesenchymal Stromal Cell-Mediated IMMUNE SUPPRESSION

\begin{tabular}{lcrc}
\hline Gene & References & Gene & References \\
\hline HMOX1 & 69 & LIF & 25 \\
TNFAIP6 & 70 & IL-6 & 25 \\
ICAM2 & 71 & IL-10 & 69 \\
NOS1 & 25 & IL-11 & 25 \\
NOS2 & 25 & VCAM1 & 23 \\
NOS3 & 25 & CXCL9 & 71 \\
PTGS1 & 71 & CXCL10 & 71 \\
PTGS2 & 71 & ACKR3 & 71 \\
PTGDS & 71 & CCL8 & 71 \\
IDO1 & 25 & LGALS1 & 72 \\
IDO2 & 25 & LGALS3 & 73 \\
TGF- $\beta 1$ & 25 & HGF & 25 \\
\hline
\end{tabular}

The expression levels of 24 genes that have been reported to be involved in MSC-mediated suppression of PBMCs (Table 1) were then examined (Fig. 5). Seven of these genes were significantly more highly expressed in adult and/or fetal tenocytes compared with ESC-tenocytes, but interestingly five of the genes were expressed at significantly lower levels in adult and/ or fetal tenocytes than ESC-tenocytes.

\section{Discussion}

We have previously derived [13] and characterized [14] equine ESCs, and demonstrated that they can differentiate into tenocytes in vitro $[16,18,19,33]$ and in vivo $[15,16]$. To be useful therapeutically as a source of tenocytes to improve tendon regeneration postinjury, the cells must avoid recognition by the recipient animal's immune system. We have previously demonstrated that equine ESCs may be immune privileged in vitro [20] and in vivo [15]. There are conflicting data on the immune properties of ESCs from other species, with some reports demonstrating that undifferentiated and differentiated ESCs are immune privileged [48-53], and

PANTHER GO-Slim Biological Process

Count Input (fold Enrichment) Input (raw P-value) Input (FDR)

Extracellular matrix organization (GO:0030198)

Extracellular structure organization (GO:0043062)

Multicellular organism development (GO:0007275)

Signal transduction (GO:0007165)

Developmental process (GO:0032502)

Cell-cell signaling (GO:0007267)

Cell communication (GO:0007154)

Cell surface receptor signaling pathway (GO:0007166)

System development (GO:0048731)

Enzyme linked receptor protein signaling pathway (GO:0007167)

Cellular response to stimulus (GO:0051716)

Transmembrane receptor protein tyrosine kinase signaling pathway (GO:0007169)

Multicellular organismal process (GO:0032501)

Cell-cell signaling by wnt (GO:0198738)

Biological adhesion (GO:0022610)

\begin{tabular}{c|l|r|r|r}
\hline 29 & 6.1 & $1.70 \mathrm{E}-12$ & $7.57 \mathrm{E}-10$ \\
29 & & 5.85 & $3.89 \mathrm{E}-12$ & $1.39 \mathrm{E}-09$ \\
90 & & 2.27 & $1.80 \mathrm{E}-11$ & $5.35 \mathrm{E}-09$ \\
180 & & 1.7 & $4.13 \mathrm{E}-11$ & $1.05 \mathrm{E}-08$ \\
68 & & 2.53 & $1.15 \mathrm{E}-10$ & $2.57 \mathrm{E}-08$ \\
76 & & 2.32 & $3.08 \mathrm{E}-10$ & $6.11 \mathrm{E}-08$ \\
76 & & 2.3 & $3.93 \mathrm{E}-10$ & $7.01 \mathrm{E}-08$ \\
87 & & 2.1 & $1.82 \mathrm{E}-09$ & $2.95 \mathrm{E}-07$ \\
68 & & 2.18 & $3.35 \mathrm{E}-08$ & $4.98 \mathrm{E}-06$ \\
43 & & 2.78 & $4.26 \mathrm{E}-08$ & $5.85 \mathrm{E}-06$ \\
186 & & 1.49 & $1.62 \mathrm{E}-07$ & $1.93 \mathrm{E}-05$ \\
36 & & 2.88 & $1.59 \mathrm{E}-07$ & $2.02 \mathrm{E}-05$ \\
163 & & 1.53 & $3.48 \mathrm{E}-07$ & $3.88 \mathrm{E}-05$ \\
22 & & 3.77 & $9.80 \mathrm{E}-07$ & $8.32 \mathrm{E}-05$ \\
50 & & 2.26 & $9.37 \mathrm{E}-07$ & $8.36 \mathrm{E}-05$ \\
\hline
\end{tabular}

FIG. 4. Gene ontology analysis of genes, which are expressed at significantly higher levels in both fetal and adult tenocytes than ESC-tenocytes Panther was used to determine the biological processes, which were over-represented by the differentially expressed genes. The top 15 enriched terms ranked by significance (FDR) are shown. FDR, false discovery rate. Color images are available online. 
Genes Involved in Modulation Inflammation
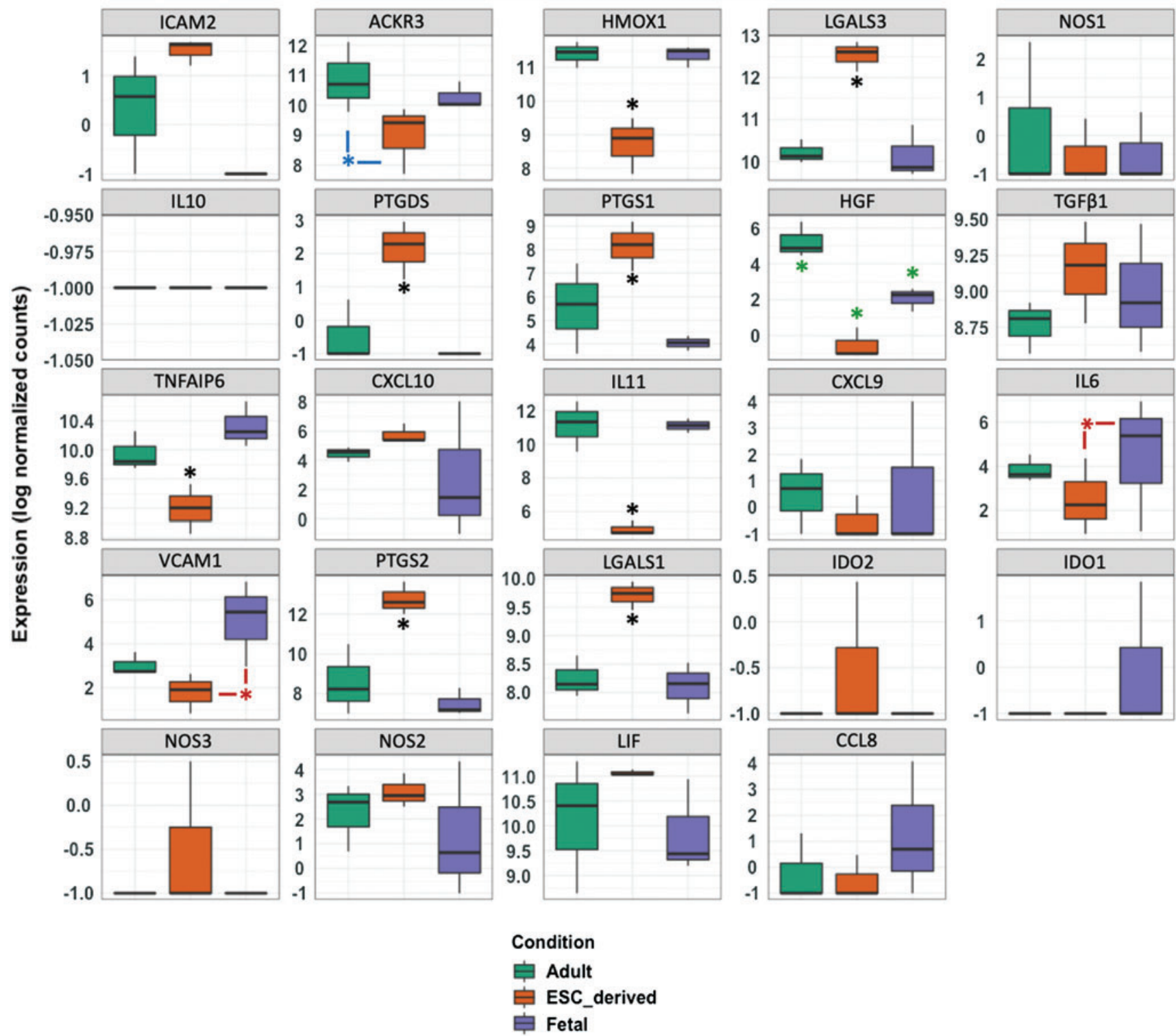

FIG. 5. Genes that are associated with MSC-mediated suppression of PBMCs exhibit variable expression between adult/ fetal tenocytes and ESC-tenocytes. Box and whisker plots showing the log normalized counts of genes in adult tenocytes (green), fetal tenocytes ( purple), and ESC-tenocytes (orange). $* P_{\text {adj }}<0.05$. black $*=$ expression significantly different in the ESC-tenocytes compared with both fetal and adult tenocytes, blue $*=$ expression only significantly different between the adult and ESC-tenocytes, red $*=$ expression only significantly different between the fetal and ESC-tenocytes, green $*=$ expression different between all three groups. MSC, mesenchymal stromal cell. Color images are available online.

others suggesting that they can still produce an immune response [54-56]. It is likely that this, in part, reflects the effect of different tissue environments into which the cells are placed [57,58]. The immune properties of specific, therapeutically relevant cell types such as tenocytes derived from ESCs remain unknown, with the exception of ESC-derived MSCs, which have been shown to be immune suppressive [59].

Although allogeneic tendon tissue transplantations are carried out in humans [11], they require decellularization to remove the immune cells within the tissue to prevent an immune response [12]. Tendon-derived stem cells have been shown to be nonimmunogenic in vitro [60] and in vivo [61,62], and MSCs stimulated to differentiate toward teno- cytes have been successfully used in allogeneic transplantations in horses [63]. However, to our knowledge, there are no reports on the immune properties of tenocytes isolated from tendon tissue.

In this study, we determined the immunogenic and immune modulatory properties of ESC-derived tenocytes in comparison with tenocytes derived from healthy adult and fetal tendon tissues. Differentiation of the ESCs into tenocytes was performed in $2 \mathrm{D}$ culture using TGF- $\beta 3$. We have previously demonstrated that this results in the expression of a variety of tendon-associated genes and proteins [16], with $41 \%$ of the cells expressing the mature tenocyte marker tenomodulin [33]. We have not yet been able to successfully 
live sort these cells, and therefore acknowledge that these experiments were carried out on a heterogeneous population of cells.

After a tendon injury, IFN $\gamma, \mathrm{TNF} \alpha$, and IL-1 $\beta$ have all been reported to be upregulated [32]. IFN $\gamma$ has previously been shown to upregulate MHC I expression on equine MSCs [20,64,65], human MSCs [66], and equine ESCs [20]. Here, we demonstrate that adult, fetal, and ESC-tenocytes express no detectable MHC II and while MHC I was not detected in adult and fetal tenocytes, the ESC-tenocytes expressed a low, heterogeneous level of MHC I, which may reflect the heterogeneity of the culture after differentiation. IFN $\gamma$ strongly increases the expression of MHC I on tenocytes derived from adult, fetal, and ESC sources, but fails to induce the expression of MHC II. Flow cytometry was not used in this study, and so the results for the MHC expression are not quantitative; however, it appears from the immunocytochemical results that MHC I is induced in every cell after $72 \mathrm{~h}$ of IFN $\gamma$ treatment in adult, fetal, and ESCtenocytes. In contrast, MHC I and MHC II remained undetectable when adult and fetal tenocytes were exposed to $\mathrm{TNF} \alpha$ or IL- $\beta$. Therefore, only IFN $\gamma$ was used in the subsequent experiments performed during this study.

In this study, we demonstrate that adult, fetal, and ESCderived tenocytes do not induce PBMC proliferation, even in the presence of IFN $\gamma$. This suggests that the upregulation of MHC I in the presence of IFN $\gamma$ does not affect allorecognition by the PBMCs, similar to results with MSCs $[20,66]$. We further show that adult and fetal tenocytes produce factors that suppress the proliferation of PHAactivated PBMCs at a 1:5 ratio tenocytes:PBMCs. This is irrespective of whether the cells have been exposed to IFN $\gamma$. In contrast, the ESC-derived tenocytes fail to suppress the proliferation of PHA-activated PBMCs at a 1:5 ratio. However, as ESCs differentiated in 2D culture only produce $\sim 40 \%$ of cells, which express the tenocyte marker tenomodulin [33], we also tested the effect of using a 1:2.5 ratio of ESC-tenocytes to PBMCs. This gave low levels of suppression $(20 \%-30 \%)$, but this was only significant in the presence of IFN $\gamma$.

To our knowledge, this is the first report to show that allogeneic tenocytes do not illicit PBMC proliferation and can suppress activated PBMCs. However, the finding is supported by numerous publications, which demonstrate that fibroblasts from other tissue sources have immune modulatory properties [37-43]. These results challenge the notion that immune modulation is a property specific to MSCs, and the current criteria for the classification of MSCs [67] do not distinguish them from fibroblasts [37]. In this study, we did not directly compare the levels of immune suppression produced by tenocytes with equine MSCs, nor did we determine if tenocytes produce suppressive factors constitutively (eg, by using conditioned media) [20], or if these factors are only produced in response to coculture with PBMCs or chemical stimulation with PHA [21].

Upon activation, equine PBMCs upregulate IFN $\gamma$ production [20,21], which can prime MSCs to become immune suppressive [68]. The addition of exogenous IFN $\gamma$ has previously been shown to increase immune suppression by MSCs [66], but in our experiments it did not appear to increase the suppression of activated PBMCs by adult and fetal tenocytes. However, a high level of suppression (up to $80 \%$ ) was produced in the absence of IFN $\gamma$, and to determine if exogenously applied IFN $\gamma$ has any additional effect it may be necessary to use a higher ratio of tenocytes to PBMCs to reduce the level of suppression produced by non-IFN $\gamma$ treated tenocytes.

Interestingly, ESC-tenocytes were only able to produce a significant level of suppression when the coculture with activated PBMCs was performed in the presence of IFN $\gamma$. We have previously shown that ESC-derived tenocytes are resistant to another inflammatory cytokine, IL-1 $\beta$, through their differential expression of IL- $1 \beta$ receptors [ie, low expression of IL-1 receptor 1 and high expression of the decoy receptor IL-1 receptor 2 and IL-1 receptor antagonist protein (IL-1Ra)] [33]. In contrast, here we find that the ESC-tenocytes express significantly higher levels of the genes encoding the IFN $\gamma$ receptors, IFNGRI and IFNGR2, than adult and fetal tenocytes, and that tenocytes from all sources are responsive to IFN $\gamma$ signaling. Equine MSCs treated with IFN $\gamma$ increase their expression of some genes involved in suppression, including IDO, PTGS2, IL-6, CXCL1O [64], and further work is needed to determine if IFN $\gamma$ increases the expression of immune modulatory genes in ESC-tenocytes.

It is not yet clear why ESC-tenocytes are apparently less immune suppressive than adult and fetal tenocytes. RNA sequencing performed on adult, fetal, and ESC-tenocytes in 2D culture demonstrated that genes that were expressed at significantly higher levels in adult and fetal tenocytes than the ESC-tenocytes were associated with a wide range of biological processes. When the relative expression levels of 24 genes involved in immune modulation by MSCs were examined, it was found that although 7 of them were expressed at higher levels in fetal and/or adult tenocytes, 5 genes were actually expressed at significantly higher levels in the ESC-tenocytes. However, these data were using nonstimulated tenocytes in regular 2D culture. It is possible that the ESC-tenocytes may represent a less mature tendon cell state, which has lower immune modulation potential, but it is also possible that the adult and fetal cells react more strongly to activation signals produced by PBMCs and/or PHA to become immune modulatory. Determining the gene expression profiles of adult, fetal, and ESC-tenocytes in response to coculture and PHA stimulation would be required to help differentiate between these possibilities.

Overall, our results suggest that allogeneic tenocytes derived from adult and fetal tendon tissues and ESCs would be safe to use in clinical transplantations in vivo. The clinical relevance of the immune suppressive properties of the tenocytes is yet to be determined. Although we found that fetal and adult tenocytes have similar immune modulatory properties, fetal tenocytes have been shown to produce better tendon tissue regeneration than adult tenocytes in a mouse model of tendon injury [9]. This suggests that the cells are using other mechanisms of action to aid tissue repair in addition to immune modulation, and the lower immune modulation by ESC-tenocytes may not have functional relevance to tissue regeneration in vivo.

\section{Acknowledgments}

The authors thank the Animal Health Trust Estate team for collecting the blood samples from the ponies. 


\section{Data Availability}

Sequence data generated in this study have been submitted to National Center for Biotechnology Information Gene Expression Omnibus (NCBI GEO, www.ncbi.nlm .nih.gov/geo) under accession number GSE132358. Differential expression analysis and normalized counts for all genes and samples are included in Supplementary Table S1.

\section{Author Disclosure Statement}

No competing financial interests exist.

\section{Funding Information}

This study was funded by the Horserace Betting Levy Board (vet/prj/782) and the Racing Foundation.

\section{Supplementary Material}

Supplementary Table S1

Supplementary Table S2

\section{References}

1. Williams RB, LS Harkins and JLN Wood. (2001). Racehorse injuries, clinical problems and fatalities recorded on British racecourses from flat racing and National Hunt racing during 1996, 1997 and 1998. Equine Vet J 33:478-486.

2. Kannus P. (1997). Tendons-a source of major concern in competitive and recreational athletes. Scand J Med Sci Sports 7:53-54.

3. Spaas JH, DJ Guest and GR Van de Walle. (2012). Tendon regeneration in human and equine athletes: Ubi Sumus-Quo Vadimus (where are we and where are we going to)? Sports Med 42:871-890.

4. Patterson-Kane JC and T Rich. (2014). Achilles tendon injuries in elite athletes: lessons in pathophysiology from their equine counterparts. ILAR J 55:86-99.

5. Dyson SJ. (2004). Medical management of superficial digital flexor tendonitis: a comparative study in 219 horses (1992-2000). Equine Vet J 36:415-419.

6. Lam KH, TDH Parkin, CM Riggs and KL Morgan. (2007). Descriptive analysis of retirement of Thoroughbred racehorses due to tendon injuries at the Hong Kong Jockey Club (1992-2004). Equine Vet J 39:143-148.

7. Beredjiklian PK, M Favata, JS Cartmell, CL Flanagan, TM Crombleholme and LJ Soslowsky. (2003). Regenerative versus reparative healing in tendon: a study of biomechanical and histological properties in fetal sheep. $J$ Biomed Eng 31:1143-1152.

8. Favata M, PK Beredjiklian, MH Zgonis, DP Beason, TM Crombleholme, AF Jawad and LJ Soslowsky. (2006). Regenerative properties of fetal sheep tendon are not adversely affected by transplantation into an adult environment. J Orthop Res 24:2124-2132.

9. Tang QM, JL Chen, WL Shen, Z Yin, HH Liu, Z Fang, BC Heng, HW Ouyang and X Chen. (2014). Fetal and adult fibroblasts display intrinsic differences in tendon tissue engineering and regeneration. Sci Rep 4:5515.

10. Bach BR, Jr., S Tradonsky, J Bojchuk, ME Levy, CA BushJoseph and NH Khan. (1998). Arthroscopically assisted anterior cruciate ligament reconstruction using patellar tendon autograft. Five- to nine-year follow-up evaluation. Am J Sports Med 26:20-29.
11. Longo UG, A Lamberti, N Maffulli and V Denaro. (2010). Tendon augmentation grafts: a systematic review. Br Med Bull 94:165-188.

12. Minami A, M Usui, S Ishii and H Kobayashi. (1983). The in vivo effects of various immunoreactive treatments on allogeneic tendon grafts. J Hand Surg Am 8:888-893.

13. Li X, SG Zhou, MP Imreh, L Ahrlund-Richter and WR Allen. (2006). Horse embryonic stem cell-like cell lines from the proliferation of inner cell mass cells. Stem Cells Dev 15:523-531.

14. Guest DJ and WR Allen. (2007). Expression of cell surface antigens and embryonic stem cell pluripotency genes in equine blastocysts. Stem Cells Dev 16:789-795.

15. Guest DJ, MRW Smith and WR Allen. (2010). Equine embryonic stem-like cells and mesenchymal stromal cells have different survival rates and migration patterns following their injection into damaged superficial digital flexor tendons. Equine Vet J 42:636-642.

16. Barsby T and D Guest. (2013). Transforming growth factor beta3 promotes tendon differentiation of equine embryoderived stem cells. Tissue Eng Part A 19:2156-2165.

17. Becerra P, M Valdes Vaquez, J Dudhia, AR Fiske-Jackson, F Neves, NG Hartman and RKW Smith. (2013). Distribution of injected technetium ${ }^{99 m}$ labeled mesenchymal stem cells in horses with naturally occuring tendinopathy. J Orthop Res 31:1096-1102.

18. Barsby T, EP Bavin and DJ Guest. (2014). Threedimensional culture and transforming growth factor beta3 synergistically promote tenogenic differentiation of equine embryo-derived stem cells. Tissue Eng Part A 20:26042613.

19. Bavin EP, F Atkinson, T Barsby and DJ Guest. (2017). Scleraxis is essential for tendon differentiation by equine embryonic stem cells and in equine fetal tenocytes. Stem Cells Dev 26:441-450.

20. Paterson Y, N Rash, E Garvican, R Paillot and DJ Guest. (2014). Equine mesenchymal stromal cells and embryoderived stem cells are immune privileged in vitro. Stem Cell Res Ther 5:90.

21. Carrade DD, MW Lame, MS Kent, KC Clark, NJ Walker and DL Borjesson. (2012). Comparative analysis of the immunomodulatory properties of equine adult-derived mesenchymal stem cells. Cell Med 4:1-11.

22. Carrade Holt DD, JA Wood, JL Granick, NJ Walker, KC Clark and DL Borjesson. (2014). Equine mesenchymal stem cells inhibit $\mathrm{T}$ cell proliferation through different mechanisms depending on tissue source. Stem Cells Dev 23:1258-1265.

23. Barrachina L, AR Remacha, A Romero, FJ Vazquez, J Albareda, M Prades, J Gosalvez, R Roy, P Zaragoza, I Martin-Burriel and C Rodellar. (2017). Priming equine bone marrow-derived mesenchymal stem cells with proinflammatory cytokines: implications in immunomodulationimmunogenicity balance, cell viability, and differentiation potential. Stem Cells Dev 26:15-24.

24. Aggarwal S and MF Pittenger. (2005). Human mesenchymal stem cells modulate allogeneic immune cell responses. Blood 105:1815-1822.

25. Gao F, SM Chiu, DA Motan, Z Zhang, L Chen, HL Ji, HF Tse, QL Fu and Q Lian. (2016). Mesenchymal stem cells and immunomodulation: current status and future prospects. Cell Death Dis 7:e2062.

26. Dakin SG, D Werling, A Hibbert, DR Abayasekara, NJ Young, RK Smith and J Dudhia. (2012). Macrophage sub- 
populations and the lipoxin A4 receptor implicate active inflammation during equine tendon repair. PLoS One 7: e32333.

27. Dakin SG, J Dudhia, NJ Werling, D Werling, DR Abayasekara and RK Smith. (2012). Inflamm-aging and arachadonic acid metabolite differences with stage of tendon disease. PLoS One 7:e48978.

28. Millar NL, GA Murrell and IB McInnes. (2017). Inflammatory mechanisms in tendinopathy-towards translation. Nat Rev Rheumatol 13:110-122.

29. Rees JD, M Stride and A Scott. (2014). Tendons-time to revisit inflammation. Br J Sports Med 48:1553-1557.

30. Dakin SG, J Dudhia and RK Smith. (2013). Science in brief: resolving tendon inflammation. A new perspective. Equine Vet J 45:398-400.

31. Clegg PD, S Strassburg and RK Smith. (2007). Cell phenotypic variation in normal and damaged tendons. Int J Exp Path 88:227-235.

32. Hosaka Y, R Kirisawa, E Yamamoto, H Ueda, H Iwai and $\mathrm{K}$ Takehana. (2002). Localisation of cytokines in tendinocytes of the superficial digital flexor tendon in the horse. $\mathrm{J}$ Vet Med Sci 64:945-947.

33. McClellan A, R Evans, C Sze, S Kan, Y Paterson and D Guest. (2019). A novel mechanism for the protection of embryonic stem cell derived tenocytes from inflammatory cytokine interleukin 1 beta. Sci Rep 9:2755.

34. Carrade DD and DL Borjesson. (2013). Immunomodulation by mesenchymal stem cells in veterinary species. Comp Med 63:207-217.

35. Caplan AI. (2017). Mesenchymal stem cells: time to change the name! Stem Cells Transl Med 6:1445-1451.

36. Caplan AI and JE Dennis. (2006). Mesenchymal stem cells as trophic mediators. J Cell Biochem 98:1076-1084.

37. Denu RA, S Nemcek, DD Bloom, AD Goodrich, J Kim, DF Mosher and P Hematti. (2016). Fibroblasts and mesenchymal stromal/stem cells are phenotypically indistinguishable. Acta Haematol 136:85-97.

38. Bouffi C, C Bony, C Jorgensen and D Noël. (2011). Skin fibroblasts are potent suppressors of inflammation in experimental arthritis. Ann Rheum Dis 70:1671-1676.

39. Korn JH. (1981). Modulation of lymphocyte mitogen responses by cocultured fibroblasts. Cell Immunol 63:374-384.

40. Shimabukuro Y, S Murakami and H Okada. (1992). Interferon-gamma-dependent immunosuppressive effects of human gingival fibroblasts. Immunology 76:344-347.

41. Donnelly JJ, MS Xi and JH Rockey. (1993). A soluble product of human corneal fibroblasts inhibits lymphocyte activation. Enhancement by interferon-gamma. Exp Eye Res 56:157-165.

42. Le JM and J Vilcek. (1987). Accessory function of human fibroblasts in mitogen-stimulated interferon-gamma production by $\mathrm{T}$ lymphocytes. Inhibition by interleukin 1 and tumor necrosis factor. J Immunol 139:3330-3337.

43. Haniffa MA, MP Collin, CD Buckley and F Dazzi. (2009). Mesenchymal stem cells: the fibroblasts' new clothes? Haematologica 94:258-263.

44. Paillot R, L Prowse, F Montesso, CM Huang, H Barnes and J Escala. (2013). Whole inactivated equine influenza vaccine: efficacy against a representative clade 2 equine influenza virus, IFNgamma synthesis and duration of humoral immunity. Vet Microbiol 162:396-407.

45. Patro R, G Duggal, MI Love, RA Irizarry and C Kingsford. (2017). Salmon provides fast and bias-aware quantification of transcript expression. Nat Methods 14:417-419.
46. Soneson C, MI Love and MD Robinson. (2015). Differential analyses for RNA-seq: transcript-level estimates improve gene-level inferences. F1000Res 4:1521.

47. Love MI, W Huber and S Anders. (2014). Moderated estimation of fold change and dispersion for RNA-seq data with DESeq2. Genome Biol 15:550.

48. Mohib K, B Alkhamees, HS Zein, D Allan and L Wang. (2012). Embryonic stem cell-derived factors inhibit T effector activation and inudce $\mathrm{T}$ regulatory cells by suppressing PKC- $\theta$ activation. PLoS One 7:e3420.

49. Mohib K, D Allan and L Wang. (2010). Human embryonic stem cell-extracts inhibit the differentiation and function of monocyte-derived dendritic cells. Stem Cell Rev Rep 6: 611-621.

50. Koch CA, P Geraldes and JL Platt. (2008). Immunosuppression by embryonic stem cells. Stem Cells 26:89-98.

51. Drukker M, H Katchman, G Katz, S Even-Tov Friedman, E Shezen, E Hornstein, O Mandelboim, Y Reisner and N Benvenisty. (2006). Human embryonic stem cells and their differentiated derivatives are less susceptible to immune rejection than adult cells. Stem Cells 24:221-229.

52. Fandrich F, B Dresske, M Bader and M Schulze. (2002). Embryonic stem cells share immune-privileged features relevant for tolerance induction. J Mol Med 80:343-350.

53. Li L, ML Baroja, A Majumdar, K Chadwick, A Rouleau, L Gallacher, I Ferber, J Lebkowski, T Martin, J Madrenas and M Bhatia. (2004). Human embryonic stem cells possess immune-privileged properties. Stem Cells 22:448456.

54. Fairchild PJ, NJ Robertson, SL Minger and H Waldmann. (2007). Embryonic stem cells: protecting pluripotency from alloreactivity. Curr Opin Immunol 19:596-602.

55. Robertson NJ, FA Brook, RL Gardner, SP Cobbold, H Waldmann and PJ Fairchild. (2007). Embryonic stem cellderived tissues are immunogenic but their inherent immune privilege promotes the induction of tolerance. Proc Natl Acad Sci U S A 104:20920-20925.

56. Yuan X, H Zhang, Y Wei and S Hu. (2007). Embryonic stem cell transplantation for the treatment of myocardial infarction: immune privilege or rejection. Transpl Immunol 18:88-93.

57. Bifari F, L Pacelli and M Krampera. (2010). Immunological properties of embryonic and adult stem cells. World J Stem Cells 2:50-60.

58. Drukker M. (2008). Immunological Considerations for Cell Therapy Using Human Embryonic Stem Cell Derivatives. Stem Book, Cambridge.

59. Yen BL, CJ Chang, K-J Liu, YC Chen, H-I Hu, C-H Bai and M-L Yen. (2009). Brief report-human embryonic stem cell-derived mesenchymal progenitors possess strong immunosuppressive effects toward natural killer cells as well as T lymphocytes. Stem Cells 27:451-456.

60. Lui PP, SK Kong, PM Lau, YM Wong, YW Lee, C Tan and OT Wong. (2014). Immunogenicity and escape mechanisms of allogeneic tendon-derived stem cells. Tissue Eng Part A 20:3010-3020.

61. Shen W, J Chen, Z Yin, X Chen, H Liu, BC Heng, W Chen and HW Ouyang. (2012). Allogenous tendon stem/ progenitor cells in silk scaffold for functional shoulder repair. Cell Transplant 21:943-958.

62. Lui PP, SK Kong, PM Lau, YM Wong, YW Lee, C Tan and OT Wong. (2014). Allogeneic tendon-derived stem cells promote tendon healing and suppress immunoreactions in hosts: in vivo model. Tissue Eng Part A 20:2998-3009. 
63. Beerts C, M Suls, SY Broeckx, B Seys, A Vandenberghe, J Declercq, L Duchateau, MA Vidal and JH Spaas. (2017). Tenogenically induced allogeneic peripheral blood mesenchymal stem cells in allogeneic platelet-rich plasma: 2year follow-up after tendon or ligament treatment in horses. Front Vet Sci 4:158.

64. Cassano JM, LA Fortier, RB Hicks, RM Harman and GR Van de Walle. (2018). Equine mesenchymal stromal cells from different tissue sources display comparable immune-related gene expression profiles in response to interferon gamma (IFN)-gamma. Vet Immunol Immunopathol 202:25-30.

65. Schnabel LV, LM Pezzanite, DF Antczak, MJ Felippe and LA Fortier. (2014). Equine bone marrow-derived mesenchymal stromal cells are heterogeneous in MHC class II expression and capable of inciting an immune response in vitro. Stem Cell Res Ther 5:13.

66. Prasanna SJ, D Gopalakrishnan, SR Shankar and AB Vasandan. (2010). Pro-inflammatory cytokines, IFN $\gamma$ and $\mathrm{TNF} \alpha$, influence immune properties of human bone marrow and Wharton jelly mesenchymal stem cells differentially. PLoS One 5:e9016.

67. Dominici M, K Le Blanc, I Mueller, I Slaper-Cortenbach, FC Marini, DS Krause, RJ Deans, A Keating, DJ Prockop and EM Horwitz. (2006). Minimal criteria for defining multipotent mesenchymal stromal cells. The International Society for Cellular Therapy position statement. Cytotherapy 8:315-317.

68. Shi Y, J Su, AI Roberts, P Shou, AB Rabson and G Ren. (2012). How mesenchymal stem cells interact with tissue immune responses. Trends Immunol 33:136-143.

69. Volarevic V, M Gazdic, B Simovic Markovic, N Jovicic, V Djonov and N Arsenijevic. (2017). Mesenchymal stem cellderived factors: immuno-modulatory effects and therapeutic potential. Biofactors 43:633-644.
70. Lee RH, JM Yu, AM Foskett, G Peltier, JC Reneau, N Bazhanov, JY Oh and DJ Prockop. (2014). TSG-6 as a biomarker to predict efficacy of human mesenchymal stem/ progenitor cells (hMSCs) in modulating sterile inflammation in vivo. Proc Natl Acad Sci U S A 111:16766-16771.

71. Lee MW, S Ryu, DS Kim, KW Sung, HH Koo and KH Yoo. (2015). Strategies to improve the immunosuppressive properties of human mesenchymal stem cells. Stem Cell Res Ther 6:179.

72. Sioud M, A Mobergslien, A Boudabous and Y Floisand. (2011). Mesenchymal stem cell-mediated T cell suppression occurs through secreted galectins. Int J Oncol 38:385390.

73. Sioud M, A Mobergslien, A Boudabous and Y Floisand. (2010). Evidence for the involvement of galectin-3 in mesenchymal stem cell suppression of allogeneic T-cell proliferation. Scand J Immunol 71:267-274.

Address correspondence to:

Dr. Deborah Jane Guest

Centre for Preventive Medicine

Animal Health Trust

Lanwades Park

Kentford

Newmarket

CB8 7UU Suffolk

United Kingdom

E-mail: debbie.guest@aht.org.uk

Received for publication June 10, 2019

Accepted after revision September 10, 2019

Prepublished on Liebert Instant Online September 11, 2019 